\title{
Intraoperative Hemorrhage, CTCAE
}

National Cancer Institute

\section{Source}

National Cancer Institute. Intraoperative Hemorrhage, CT CAE. NCI Thesaurus. Code C143604.

A finding of uncontrolled bleeding during a surgical procedure. 